\title{
On The Para-Octonions; a Non-Associative Normed Algebra
}

\author{
Mehdi JAFARI \\ Department of Mathematics, Technical and Vocational University, Urmia, Iran
}

\begin{abstract}
ABSTARCT
In this paper, para-octonions and their algebraic properties are provided by using the Cayley-Dickson multiplication rule between the octonionic basis elements. The trigonometric form of a para-octonion is similar to the trigonometric form of dual number and quasi-quaternion. We study the De-Moivre's theorem for para-octonions, extending results obtained for real octonions and defining generalize Euler's formula for para-octonions.
\end{abstract}

Keywords: Alternativity, Cayley-Dickson construction, De-Moivre's formula, Para-octonion

\section{Para-Ktonyonlar Üzerine; Bir İlişkisel Olmayan Normlu Cebir}

\section{ÖZET}

Bu çalışmada, octonyonik baz elemanları arasında Cayley-Dickson çarpım kuralı kullanılarak para-octonyonlar ve cebirsel özellikleri verilmiştir. Bir para-octonyonun trigonometrik formu bir dual-sayının ve bir quasi-kuaterniyonun trigonometrik formuna benzerdir. Para-octonyonlar içn De-Moivre'nin teoremi ele alınarak reel-octonyonlar için elde edilen sonuçlar genelleştirilmiştir. Ayrıca, para-octonyonlar için genel Euler formülleri tanımlanmıştır.

Anahtar kelimeler: Alternatiflik, Cayley-Dickson yap1, De-Moivre formu, para-oktoniyon

\section{INTRODUCTION}

The real octonions algebra as the ordered couple of real quaternions, was invented by J. T. Graves (1843) and A. Cayley (1845) independently. In mathematics, the real octonions form a normed division algebra over the real numbers, usually represented by $\mathrm{O}$. In our previous works, we studied some algebraic properties of real, split, complex, semi-octonions, and quasi-octonions.

In this paper, we study some algebraic properties of para-octonions, which is called $1 / 8-$ octonions in [9]. A pare-octonions can be written in form a dual quasi-quaternions. We review the generalized octonions algebra, and show that if put $\alpha=\beta=\gamma=0$, we obtain para-octonions algebra. Like real octonions, para-octonions form a non-associative algebra, but unlike real octonions, they are not division algebra. We investigate the De Moivre's formula for these octonions and by using this formula, we obtain any power of a para-octonion. We hope that this work will contribute to the study of physics and other sciences.

\section{THEORETICAL BACKGROUND}

In this section, we give a brief summary of the generalized octonions. For detailed information about these octonions, we refer the reader to [1].

Definition 1. A generalized octonion $x$ is defined as $x=a_{0} e_{0}+a_{1} e_{1}+a_{2} e_{2}+a_{3} e_{3}+a_{4} e_{4}+a_{5} e_{5}+a_{6} e_{6}+a_{7} e_{7}$, where $a_{0}, \ldots, a_{7}$ are real numbers and $e_{i},(0 \leq i \leq 7)$ are octonionic units satisfying the equalities that are given in the following table; 
The multiplication rules among the basis elements of octonions $e_{i}$ can be expressed in the form:

\begin{tabular}{|c|c|c|c|c|c|c|c|}
\hline$\cdot$ & $e_{1}$ & $e_{2}$ & $e_{3}$ & $e_{4}$ & $e_{5}$ & $e_{6}$ & $e_{7}$ \\
\hline$e_{1}$ & $-\alpha$ & $e_{3}$ & $-\alpha e_{2}$ & $e_{5}$ & $-\alpha e_{4}$ & $-e_{7}$ & $\alpha e_{6}$ \\
\hline$e_{2}$ & $-e_{3}$ & $-\beta$ & $\beta e_{1}$ & $e_{6}$ & $e_{7}$ & $-\beta e_{4}$ & $-\beta e_{5}$ \\
\hline$e_{3}$ & $\alpha e_{2}$ & $-\beta e_{1}$ & $-\alpha \beta$ & $e_{7}$ & $-\alpha e_{6}$ & $\beta e_{5}$ & $-\alpha \beta e_{4}$ \\
\hline$e_{4}$ & $-e_{5}$ & $-e_{6}$ & $-e_{7}$ & $-\gamma$ & $\gamma e_{1}$ & $\gamma e_{2}$ & $\gamma e_{3}$ \\
\hline$e_{5}$ & $\alpha e_{4}$ & $-e_{7}$ & $\alpha e_{6}$ & $-\gamma e_{1}$ & $-\alpha \gamma$ & $-\gamma e_{3}$ & $\alpha \gamma e_{2}$ \\
\hline$e_{6}$ & $e_{7}$ & $\beta e_{4}$ & $-\beta e_{5}$ & $-\gamma e_{2}$ & $\gamma e_{3}$ & $-\beta \gamma$ & $\beta \gamma$ \\
\hline$e_{7}$ & $-\alpha e_{6}$ & $\beta e_{5}$ & $\alpha \beta e_{4}$ & $-\gamma e_{3}$ & $-\alpha \gamma e_{2}$ & $\beta \gamma e_{1}$ & $-\alpha \beta \gamma$ \\
\hline
\end{tabular}

\section{Special Cases:}

1. If $\alpha=\beta=\gamma=1$, is considered, then $\mathrm{O}(\alpha, \beta, \gamma)$ is the algebra of real octonions $\mathrm{O}[5]$.

2. If $\alpha=\beta=1, \gamma=-1$, is considered, then $\mathrm{O}(\alpha, \beta, \gamma)$ is the algebra of split octonions (Psoudo-octonions) $\mathrm{O}^{\prime}[4]$.

3. If $\alpha=\beta=1, \gamma=0$, is considered, then $\mathrm{O}(\alpha, \beta, \gamma)$ is the algebra of semi-octonions $\mathrm{O}_{S}[3]$.

4. If $\alpha=\beta=-1, \gamma=0$, is considered, then $\mathrm{O}(\alpha, \beta, \gamma)$ is the algebra of split semi-octonions $\mathrm{O}_{S}^{\prime}[5]$.

5. If $\alpha=1, \beta=\gamma=0$, is considered, then $\mathrm{O}(\alpha, \beta, \gamma)$ is the algebra of quasi-octonions $\mathrm{O}_{q}$ [6].

6. If $\alpha=-1, \beta=\gamma=0$, is considered, then $\mathrm{O}(\alpha, \beta, \gamma)$ is the algebra of split quasi-octonions $\mathrm{O}_{q}^{\prime}$ [8].

7. If $\alpha=\beta=\gamma=0$, is considered, then $\mathrm{O}(\alpha, \beta, \gamma)$ is the algebra of para-octonions $\mathrm{O}_{p}$.

The generalized octonions algebra, $\mathrm{O}(\alpha, \beta, \gamma)$, is a non-commutative, non-associative, alternative, flexible and power-associative [1].

\section{PARA-OCTONIONS ALGEBRA}

Definition 2. A para-octonion $x$ is expressed as a real linear combination of the unit octonions $\left(e_{0}, e_{1}, \ldots, e_{7}\right)$, i.e.

$$
x=\left(x_{0}, x_{1}, \ldots, x_{7}\right)=x_{0} e_{0}+\sum_{i=1}^{7} x_{i} e_{i},
$$

where $x_{0}, \ldots, x_{7}$ are real numbers and $e_{i},(0 \leq i \leq 7)$ are imaginary octonion units satisfying the non-commutative multiplication rules;

$$
\begin{gathered}
e_{k}^{2}=0, \quad k=0, \ldots, 7 \\
e_{1} e_{2}=e_{3}=-e_{2} e_{1},
\end{gathered}
$$

The above multiplication rules are given in the following Table;

\begin{tabular}{|c|c|c|c|c|c|c|c|}
\hline$\cdot$ & $e_{1}$ & $e_{2}$ & $e_{3}$ & $e_{4}$ & $e_{5}$ & $e_{6}$ & $e_{7}$ \\
\hline$e_{1}$ & 0 & $e_{3}$ & 0 & $e_{5}$ & 0 & $-e_{7}$ & 0 \\
\hline$e_{2}$ & $-e_{3}$ & 0 & 0 & $e_{6}$ & $e_{7}$ & 0 & 0 \\
\hline$e_{3}$ & 0 & 0 & 0 & $e_{7}$ & 0 & 0 & 0 \\
\hline$e_{4}$ & $-e_{5}$ & $-e_{6}$ & $-e_{7}$ & 0 & 0 & 0 & 0 \\
\hline$e_{5}$ & 0 & $-e_{7}$ & 0 & 0 & 0 & 0 & 0 \\
\hline$e_{6}$ & $e_{7}$ & 0 & 0 & 0 & 0 & 0 & 0 \\
\hline$e_{7}$ & 0 & 0 & 0 & 0 & 0 & 0 & 0 \\
\hline
\end{tabular}

This form, $x=x_{0} e_{0}+\sum_{i=1}^{7} x_{i} e_{i}$, is called the standard form of a para-octonion. By using the Cayley-Dickson construction, a para-octonion $x$ can also be written as 


$$
x=\left(a_{0} e_{0}+a_{1} e_{1}+a_{2} e_{2}+a_{3} e_{3}\right)+\left(a_{4}+a_{5} e_{1}+a_{6} e_{2}+a_{7} e_{3}\right) e_{4}=q+q^{\prime} l,
$$

where $l^{2}=0$ and $q, q^{\prime}$ are quasi-quaternions (1/4 -quaternions) [2], i.e.

$$
q, q^{\prime} \in \mathrm{H}_{q}^{\circ}=\left\{q=a_{0}+a_{1} e_{1}+a_{2} e_{2}+a_{3} e_{3} \mid e_{1}^{2}=e_{2}^{2}=e_{3}^{2}=0, e_{1} e_{2}=e_{3}, e_{1} e_{3}=0=e_{2} e_{3}, a_{i} \in \mathrm{R}\right\},
$$

This construction lets us view the para-octonions as a two dimensional vector space over quasi-quaternions. A para-octonion $x$ can be decomposed in terms of its $\operatorname{scalar}\left(S_{x}\right)$ and vector $\left(\vec{V}_{x}\right)$ parts as

$$
S_{x}=a_{0}, \quad \vec{V}_{x}=a_{1} e_{1}+a_{2} e_{2}+a_{3} e_{3}+a_{4} e_{4}+a_{5} e_{5}+a_{6} e_{6}+a_{7} e_{7} .
$$

For two para-octonions $x=\sum_{i=0}^{7} a_{i} e_{i}$ and $w=\sum_{i=0}^{7} b_{i} e_{i}$, the summation and substraction processes are given as $x \pm w=\sum_{i=0}^{7}\left(a_{i} \pm b_{i}\right) e_{i}$.

The product of two para-octonions $x=S_{x}+\vec{V}_{x}, w=S_{w}+\vec{V}_{w}$ is expressed as

$$
x . w=S_{x} S_{w}-\left\langle\vec{V}_{x}, \vec{V}_{w}\right\rangle+S_{x} \vec{V}_{w}+S_{w} \vec{V}_{x}+\vec{V}_{x} \times \vec{V}_{w}
$$

This product can be described by a matrix-vector product as

$$
x . w=\left[\begin{array}{cccccccc}
a_{0} & 0 & 0 & 0 & 0 & 0 & 0 & 0 \\
a_{1} & a_{0} & 0 & 0 & 0 & 0 & 0 & 0 \\
a_{2} & 0 & a_{0} & 0 & 0 & 0 & 0 & 0 \\
a_{3} & -a_{2} & a_{1} & a_{0} & 0 & 0 & 0 & 0 \\
a_{4} & 0 & 0 & 0 & a_{0} & a_{1} & 0 & 0 \\
a_{5} & -a_{4} & 0 & 0 & a_{1} & a_{0} & 0 & 0 \\
a_{6} & 0 & -a_{4} & 0 & a_{2} & 0 & a_{0} & 0 \\
a_{7} & a_{6} & -a_{5} & -a_{4} & a_{3} & a_{2} & -a_{1} & a_{0}
\end{array}\right]\left[\begin{array}{l}
b_{0} \\
b_{1} \\
b_{2} \\
b_{3} \\
b_{4} \\
b_{5} \\
b_{6} \\
b_{7}
\end{array}\right] .
$$

Para-octonions multiplication is not associative, since

$$
\begin{aligned}
& e_{1}\left(e_{2} e_{4}\right)=e_{1} e_{6}=-e_{7}, \\
& \left(e_{1} e_{2}\right) e_{4}=e_{3} e_{4}=e_{7} .
\end{aligned}
$$

But it has the property of alternativity, that is, any two elements in it generate an associative subalgebra isomorphic to $\mathrm{R}, \mathbb{C}^{0}, \mathrm{H}^{0}$.

$e_{0}$ and $e_{i}(1 \leq i \leq 7)$ generate a subalgebra isomorphic to dual numbers $\mathbb{C}^{0}$,

Subalgebra with bases $e_{0}, e_{i}, e_{j}, e_{k}(1 \leq i, j, k \leq 7)$ is isomorphic to quasi-quaternions algebra $\mathrm{H}_{q}^{0}$.

\subsection{Some Properties of Para-octonions}

The conjugate of para-octonion $x=\sum_{i=0}^{7} a_{i} e_{i}=S_{x}+\vec{V}_{x}$ is

$$
\bar{x}=a_{0} e_{0}-\sum_{i=1}^{7} a_{i} e_{i}=S_{x}-\vec{V}_{x} .
$$

Conjugate of product of two para-octonions and its own are described as

$$
\overline{x y}=\bar{y} \bar{x}, \overline{\bar{x}}=x
$$

It is clear that the scalar and vector parts of $x$ is denoted by $S_{x}=\frac{x+\bar{x}}{2}$ and $\vec{V}_{x}=\frac{x-\bar{x}}{2}$.

1) The norm of $x$ is

$$
N_{x}=x \bar{x}=\bar{x} x=|x|^{2}=a_{0}^{2} .
$$

It satisfies the following property

$$
N_{x y}=N_{x} N_{y}=N_{y} N_{x}
$$

If $N_{x}=1$, then $x$ is called a unit para-octonion. We will use to denote the set of unit para-octonions.

2) The inverse of $x$ with $N_{x} \neq 0$, is

$$
x^{-1}=\frac{1}{N_{x}} \bar{x} .
$$

4) The trace of element $x$ is defined as $t(x)=x+\bar{x}$. 
The para-octonions algebra is not division algebra, because for every nonzero $x \in \mathrm{O}_{P}$ the relation $N_{x}=0$, implies $x \neq 0$.

Example 1. Consider the para-octonions

$$
\begin{gathered}
x_{1}=1+(1,-1,2,-2,0,1,1), \\
x_{2}=0+(1,-1,1,-2,0,1,1) \text { and } \\
x_{3}=-2+(1,-1, \sqrt{2},-2,2,1,1) ;
\end{gathered}
$$

1. The vector parts of $x_{1}, x_{2}$ are

$\vec{V}_{x_{1}}=(1,-1,2,-2,0,1,1), \vec{V}_{x_{2}}=(1,-1,1,-2,0,1,1)$.

2. The conjugates of $x_{1}, x_{2}$ are

$\bar{x}_{1}=1-(1,-1,2,-2,0,1,1), \bar{x}_{2}=0-(1,-1,1,-2,0,1,1)$.

3. The norms are given by

$$
N_{x_{1}}=1, N_{x_{2}}=0, N_{x_{3}}=4
$$

4. The inverses are

$x_{1}^{-1}=1-(1,-1,2,-2,0,1,1), \quad x_{3}^{-1}=\frac{1}{4}[-2-(1,-1, \sqrt{2},-2,2,1,1)]$, and $x_{2}$ not invertible.

5. One can realize the following operations

$$
\begin{aligned}
& x_{1}+x_{2}=1+(2,-2,3,-4,0,2,2) \\
& x_{1}-x_{2}=2+(0,0,1,0,0,0) \\
& x_{1} x_{2}=0+(1,-1,1,-2,0,1,-1) \\
& x_{2} x_{1}=0+(1,-1,1,-2,0,1,3) \\
& \quad N_{x_{1} x_{2}}=N_{x_{1}} N_{x_{2}}=N_{x_{2} x_{1}}=0 .
\end{aligned}
$$

Theorem 1.4. The set $\mathrm{O}_{P}^{1}$ of unit split semi-octonions is a subgroup of the group $\mathrm{O}_{P}^{0}$ where $\mathrm{O}_{P}^{0}=\mathrm{O}_{P}-[0-\overrightarrow{0}]$.

Proof: Let $x, y \in \mathrm{O}_{P}^{1}$. We have $N_{x y}=1$, i.e. $x y \in \mathrm{O}_{P}^{1}$ and thus the first subgroup requirement is satisfied. Also, by the property

$$
N_{x}=N_{\bar{x}}=N_{x^{-1}}=1,
$$

the second subgroup requirement $x^{-1} \in \mathrm{O}_{P}^{1}$.

\subsection{Trigonometric form and De Moivre's theorem}

The trigonometric (polar) form of a nonzero para-octonion $x=\sum_{i=0}^{7} a_{i} e_{i}$ is

$$
x=r(\cos \varphi+\vec{w} \sin \varphi)
$$

where $r=|x|=\sqrt{N_{x}}$ is the modulus of $x$,

$$
\begin{gathered}
\cos \varphi=\frac{a_{0}}{r}, \quad \sin \varphi=\varphi=\frac{\left(\sum_{i=1}^{7} a_{i}^{2}\right)^{1 / 2}}{r} \\
\text { and } \\
\vec{w}=\left(w_{1}, w_{2}, \ldots, w_{7}\right)=\frac{1}{\left(\sum_{i=1}^{7} a_{i}^{2}\right)^{1 / 2}}\left(a_{1}, a_{2}, \ldots, a_{7}\right) .
\end{gathered}
$$

This is similar to polar coordinate expression of a quasi-quaternion and dual number.

Example 2. The trigonometric forms of the para-octonions

$$
x_{1}=1+(1,-1,0,1,1,1,-1) \text { is } x_{1}=\cos \varphi+\vec{w}_{1} \sin \varphi,
$$$$
x_{2}=-2+(2,-1,0,1,-1,2,1) \text { is } x_{2}=2\left[\cos \varphi+\vec{w}_{2} \sin \varphi\right]
$$

where

$$
\begin{gathered}
\vec{w}_{1}=\frac{1}{\sqrt{6}}(1,-1,0,1,1,1,-1), \vec{w}_{2}=\frac{1}{\sqrt{12}}(2,-1,0,1,-1,2,1) \\
\text { and } N_{\vec{w}_{1}}=N_{\vec{w}_{2}}=0 .
\end{gathered}
$$

Theorem 1.5. (De Moivre's theorem) If $x=r(\cos \varphi+\vec{w} \sin \varphi$ ) be a para-octonion and $n$ is any positive integer, then

$$
x^{n}=r^{n}(\cos n \varphi+\vec{w} \sin n \varphi)
$$

Proof: The proof easily follows by induction on $n$.

The Theorem holds for all integers $n$, since

$$
\begin{gathered}
q^{-1}=\cos \varphi-\vec{w} \sin \varphi, \\
q^{-n}=\cos (-n \varphi)+\vec{w} \sin (-n \varphi) \\
=\cos n \varphi-\vec{w} \sin n \varphi .
\end{gathered}
$$

Example 3. Let $x=1+(1,-1,2,1,2,2,-1)$. Find $x^{10}$ and $x^{-45}$.

Solution: First write $x$ in trigonometric form.

$$
x=\cos \varphi+\vec{w} \sin \varphi,
$$


where $\cos \varphi=1, \sin \varphi=4, \vec{w}=1 / 4(1,-1,2,1,2,2,-1)$.

Applying de Moivre's theorem gives:

$x^{10}=\cos 10 \varphi+\vec{w} \sin 10 \varphi=1+40 \vec{w}=1+10(1,-1,2,1,2,2,-1)$

$x^{-45}=\cos (-45 \varphi)+\vec{w} \sin (-45 \varphi)=1-45(1,-1,2,1,2,2,-1)$.

Corollary 1.5. The equation for a unit para-octonion.

Example 3.5. Let $x=-1+(1,-1,2,1,1,0,-1)$ be a unit para-octonion. There is no $n(n>0)$ such that $x^{n}=1$.

For any unit para-octonion $x=\cos \varphi+\vec{w} \sin \varphi$, since $\vec{w}^{2}=0$, a natural generalization of Euler's formula is

\section{Conclusion}

In this paper, we defined and gave some of algebraic properties of para-octonions and showed that the trigonometric form of para-octonions is similar to quasi-quaternions and dual numbers. The De Moivre's formulas for these octonions is obtained. We gave some examples for clarification.

\section{Further Work}

We will give a complete investigation to real matrix representations of para-octonions, and give any powers of these matrices.

\section{REFERENCES}

$e^{\vec{w} \varphi}=1+\vec{w} \varphi+\frac{(\vec{w} \varphi)^{2}}{2 !}+\frac{(\vec{w} \varphi)^{3}}{3 !}+\ldots=1+\vec{w} \varphi=\cos \varphi+\vec{w} \sin \varphi=x,{ }^{[1]}$

\subsection{Roots of Para-octonion}

Theorem 1.6. Let $x=r(\cos \varphi+\vec{w} \sin \varphi)$ be a para-octonion. The equation $a^{n}=x$ has only one root and this is

$$
a=\sqrt[n]{r}\left(\cos \frac{\varphi}{n}+\vec{w} \sin \frac{\varphi}{n}\right)
$$

Proof: We assume that $a=M(\cos \lambda+\vec{w} \sin \lambda)$ is a root of the equation $a^{n}=x$, since the vector parts of $x$ and $a$ are the same. From Theorem 4.5, we have

Now, we find

$$
a^{n}=M^{n}(\cos n \lambda+\vec{w} \sin n \lambda) .
$$

$$
M=\sqrt[n]{r}, \quad \cos \varphi=\cos n \lambda, \quad \sin \varphi=\sin n \lambda .
$$

So, $\quad a=\sqrt[n]{r}\left(\cos \frac{\varphi}{n}+\vec{w} \sin \frac{\varphi}{n}\right)$ is a root of equation $a^{n}=x$. If we suppose that there are two roots satisfying the equality, we obtain that these roots must be equal to each other.

Example 1.6. Let $x=8+(1,0,-\sqrt{2}, 0,2,-1,0)$ be a para-octonion. The cube root of the octonion $x$ is

$$
\begin{aligned}
x^{1 / 3} & =\sqrt[3]{8}\left(\cos \frac{\varphi}{3}+\vec{w} \sin \frac{\varphi}{3}\right) \\
& =2\left(1+\frac{1}{3 \sqrt{8}} \vec{w}\right) .
\end{aligned}
$$

1] Flaut, C., \& Shpakivskyi V., (2015). An efficient method for solving equations in generalized quaternion and octonion algebras, Advance in Applied Clifford algebra, 25 (2), 337350.

[2] Jafari M., On the Properties of Quasi-Quaternions Algebra, (2014). Communications, faculty of science, university of Ankara, Series A1: Mathematics and statistics, 63(1), 1-10.

[3] Jafari M., (2015). A viewpoint on semi-octonion algebra, Journal of Selcuk university natural and applied science, 4(4), 46-53.

[4] Jafari M., (2015). Split Octonion Analysis, Representation Theory and Geometry, Submitted for publication.

[5] Jafari M., Azanchiler H., (2015). On the structure of the octonion matrices, Submitted for publication.

[6] Jafari M., (2015). An Introduction to Quasi-Octonions and Their Representation, DOI: 10.131 40/RG.2.1.3833.0082

[7] Mortazaasl H., Jafari M., (2013). A study on Semi-quaternions Algebra in Semi-Euclidean 4-Space, Mathematical Sciences and Applications E-Notes, 1(2) 20-27.

[8] Jafari M., (2015). The Fundamental Algebraic Properties of Split Quasi-Octonions, DOI: 10. 13140/RG 2.1.3348.2728

[9] Rosenfeld B. A., Geometry of Lie Groups, Kluwer Academic Publishers, Dordrecht, 1997. 